\title{
Suppression of amplitude dependent closest tune approach and its behavior under forced oscillations
}

\author{
T. Persson, ${ }^{*}$ R. Tomás, and E. H. Maclean \\ CERN, CH-1211 Geneva 23, Switzerland
}

(Received 15 March 2019; published 8 May 2019)

\begin{abstract}
An amplitude dependent closest tune approach (ADeCTA) was first observed in the LHC in 2012. This potentially harmful effect can be generated by linear coupling in conjunction with octupoles. In this article we demonstrate experimentally and in simulations a configuration of the octupoles to suppress this recently observed effect. Furthermore, ADeCTA is investigated for the first time with driven oscillations. We show analytically that skew quadrupoles and normal octupoles do not generate ADeCTA for forced oscillations in the same way as for free oscillations. This adds an additional constraint to measure it since the forced oscillations, generated by an ac dipole, are the preferred option for optics measurements in the LHC. The analytical result is confirmed by both measurements and simulations.
\end{abstract}

DOI: 10.1103/PhysRevAccelBeams.22.051001

\section{INTRODUCTION}

Experimental observations in 2012 [1] suggested the existence of an amplitude dependent closest tune approach (ADeCTA). This is a nonlinear correspondence to the linear $\left|C^{-}\right|=\Delta Q_{\min }$, but where particles of different amplitude have different $\left|C^{-}\right|$. The effect is potentially harmful since it modifies the beam spectral distribution. Simulations in [1] confirmed the existence of ADeCTA with further investigations in [2]. The key ingredients to reproduce the observations were found to be linear coupling and normal octupolar fields. Strong octupolar fields are introduced in the LHC to create Landau damping which is critical to ensure beam stability [3]. It it was also shown in [2] that normal octupoles in a coupled lattice could allow the particle tunes to penetrate the linear coupling stop band. On the theoretical side, a mechanism leading to ADeCTA has been presented in [4]. It was found that the cross amplitude detuning term $h_{1111}$ together with linear coupling generate ADeCTA, leaving open the possibility for other normal octupolar terms to contribute as well. The following equation describes the amplitude dependent $\left|C^{-}\right|$:

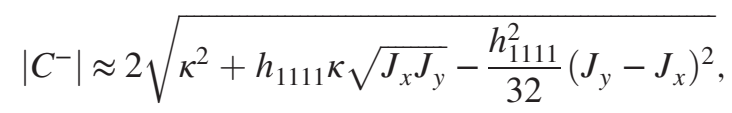

where $2|\kappa|$ is the linear $\left|C^{-}\right|, J_{x, y}$ are the action coordinates and $h_{1111}$ is given by

\footnotetext{
*tobias.persson@cern.ch
}

Published by the American Physical Society under the terms of the Creative Commons Attribution 4.0 International license. Further distribution of this work must maintain attribution to the author(s) and the published article's title, journal citation, and DOI.

$$
h_{1111}=\frac{1}{2 \pi} \int_{0}^{2 \pi} K_{3} \beta_{x} \beta_{y} d \theta
$$

where $\beta_{x, y}$ are the horizontal and vertical $\beta$-functions and $K_{3}$ is the octupole strength. The LHC is equipped with two families of octupoles used for Landau damping: magnet octupoles focusing (MOF) and magnet octupoles defocusing (MOD) [5]. The naming of the focusing and defocusing octupoles is based on whether they are placed close to a horizontal focusing or defocusing quadrupole. In Sec. II a configuration to almost cancel the $h_{1111}$, while keeping a similar level of Landau damping is described. This is achieved by having opposite polarities in the MOF and the MOD.

An ac dipole can drive the motion of the beam for thousands of turns without any emittance increase [6]. That makes it suitable for measurements in accelerators, such as the LHC, where the time to inject a new bunch and reach the configuration of interest is long. This has made the ac dipole the primary beam exciter for optics measurements $[7,8]$. It is well known that the particle motion in the presence of forced oscillations differs from the free motion following a single kick. This has led to methods which account for this difference in the motion when reconstructing the optics parameters [9-12]. However, these studies stay at linear order in the strength of perturbing components and can therefore not be used to predict the ADeCTA. In Sec. III we investigate the possibility to use forced oscillations to measure ADeCTA.

Transverse coupling has been shown to modify the impact of nonlinearities and linked to instabilities [13-15]. This has led to improve the control of the coupling in the LHC [16-20]. This might be even more important in the future since dynamic aperture studies for the LHC have 
shown that the best lifetime is obtained for tunes close to the difference coupling resonance [21]. This tighter tune split could possibly enhance unwanted effects from ADeCTA, further motivating the understanding and control of ADeCTA.

Simulations in $[4,22]$ showed that skew octupoles placed in an uncoupled lattice would not generate ADeCTA, while a combination of normal and skew octupoles in a linearly uncoupled lattice could still generate ADeCTA [22]. However, for the LHC configuration at injection, which is used throughout this article, skew octupolar components are too small to have a significant impact.

\section{MEASURING AND MITIGATING ADECTA WITH BEAM UNDER FREE OSCILLATIONS}

The experiment was done in the LHC during dedicated machine development time in 2016. The optics used was the nominal for injection and the beam energy was $450 \mathrm{GeV}$. The beam was excited with a pulsed kicker magnet [23]. The turn-by-turn data was recorded with all the $\sim 500$ available Beam Position Monitors (BPMs). After the kick the beam decohered within 200-400 turns depending on the amplitude of the kick. The measurement was truncated at the point where no more oscillations were visible and then cleaned using singular value decomposition (SVD) in order to reduce the noise and remove faulty BPMs [24]. The horizontal and vertical tunes were obtained from SUSSIX [25] and an average and standard deviation was calculated. The action was calculated from the peak amplitude divided by the $\beta$-function at the location of the BPM [1].

A key parameter to control, in order to later compare the results with simulations, is the linear coupling. The $\left|C^{-}\right|$ was increased intentionally and measured through moving the two tunes as close as possible together.

The beam is fully coupled when it is no longer possible to separate the horizontal from the vertical motion. This occurs when the two eigentunes have the smallest possible distance. In this case it is no longer possible to associate the tune of a specific plane with the corresponding eigentune. However, by measuring the distance between the two peaks it is possible to conclude on the $\left|C^{-}\right|$. In this case it was determined to be $0.0155 \pm 0.001$ [26]. The uncertainty of the measurement is less than $10^{-4}$ but a decay of the coupling has been observed at injection and this puts the error bar of 0.001 on the $\left|C^{-}\right|[18]$.

The unit used to describe the settings of the octupoles is a normalized strength, which is obtained by multiplying the magnetic field with the charge and dividing by the reference momentum [27]. The strength of a given family is written as the name of the family equal to the strength.

Initially the Landau octupoles were powered to $-5 \mathrm{~m}^{-4}$ which is stronger than the 2012 setting of $-3 \mathrm{~m}^{-4}$ but weaker and opposite polarity to the 2016 setting of $9 \mathrm{~m}^{-4}$. The amplitude for the horizontal plane kicks was kept low and constant while the amplitudes of the vertical kicks were increased. The fractional tunes for the scan were kept at $Q_{y}=0.285, Q_{x}=0.31$ and the powering of the octupoles was $\mathrm{MOF}=-5 \mathrm{~m}^{-4}, \mathrm{MOD}=-5 \mathrm{~m}^{-4}$. The second scan kept the same tunes but changed the powering of the octupoles to: $\mathrm{MOF}=5 \mathrm{~m}^{-4}, \mathrm{MOD}=-5 \mathrm{~m}^{-4}$.

The settings of the LHC were reproduced in MAD-X [28]. The coupling amplitude was generated using the arc skew quadrupoles and the strengths of the octupoles were incorporated as in the machine. No additional errors were introduced in the model. Single particles with different horizontal amplitudes and a small initial vertical amplitude $(0.5 \mathrm{~mm}$ at IP3) were tracked for 1024 turns and the same procedure, as for the measured data, was used to obtain the tunes and actions. The linear $\left|C^{-}\right|$was obtained using the same method as for the experiment, i.e. pushing the horizontal and the vertical tunes as close as possible together.

The value of the $\left|h_{1111}\right|$ was $4.2 \times 10^{4} \mathrm{~m}^{-1}$ for the equal polarity and $\left|h_{1111}\right|$ was $4.0 \times 10^{3} \mathrm{~m}^{-1}$ for the setting with opposite polarity of the two families. The value of the Hamiltonian term was obtained from the polymorphic tracking code (PTC) [29].

Figure 1 shows the tune split as a function of the vertical action for the two different configurations of the Landau octupoles described in the previous section. We observe that the fractional tune split saturates far away from the linear $\left|C^{-}\right|$. When the polarity of the MOF was changed the behavior is altered and a much more linear approach towards the $\left|C^{-}\right|$is observed. The amplitude dependent

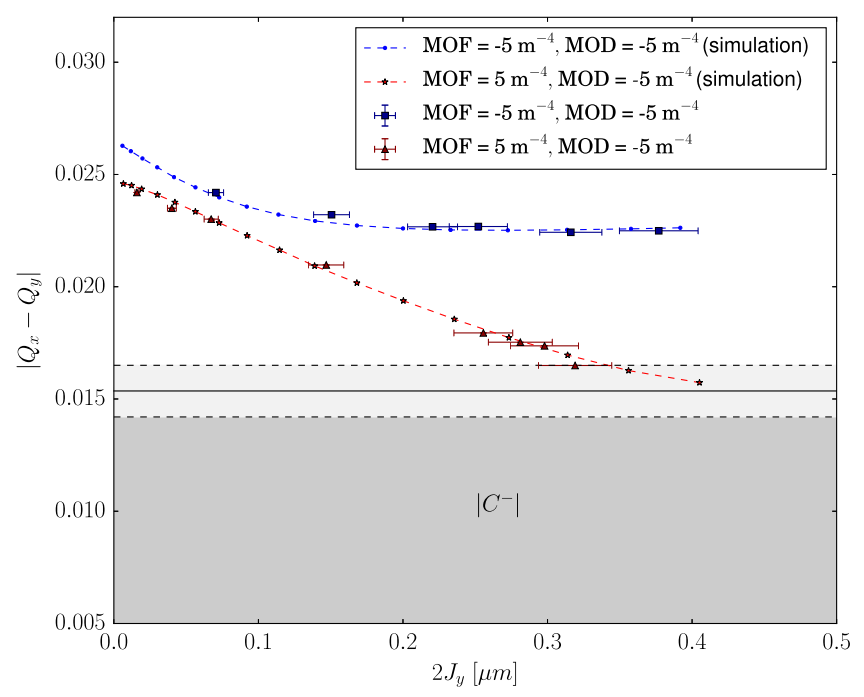

FIG. 1. The difference between the fractional horizontal and vertical tune as a function of the vertical action for free oscillations. The excitation was done using a kicker magnet and the settings of the octupoles were: $\mathrm{MOF}=-5 \mathrm{~m}^{-4}$, MOD $=$ $-5 \mathrm{~m}^{-4}$ and $\mathrm{MOF}=5 \mathrm{~m}^{-4}, \mathrm{MOD}=-5 \mathrm{~m}^{-4}$. The fractional tunes were $Q_{x}=0.285$ and $Q_{y}=0.31$. The dark gray area shows the linear $\left|C^{-}\right|$and the light gray area shows the uncertainty of the measured coupling. 
$\left|C^{-}\right|$is about $40 \%$ larger for the case with the same polarity of the two families. The dotted lines show simulations which agree well with the measured values. The experimental results are also in qualitative agreement with Eq. (1) derived in [4], given that the $\left|h_{1111}\right|$ is expected to have been a factor 10 lower for the case with opposite polarity.

\section{IMPOSSIBILITY OF MEASURING ADECTA WITH BEAM UNDER FORCED OSCILLATIONS}

In this section we investigate the possibility to measure ADeCTA using forced oscillations. This would be ideal to use for measurements at top energies since there would be no emittance increase and hence no need to reinject. The section starts with the analytical derivations and then continues with measurement and simulations.

\section{A. Theory}

In [4] ADeCTA is studied for free motion arising from a cross term between coupling and an octupolar term. In this part we investigate if ADeCTA can be generated in the same way under forced oscillations. Amplitude detuning for forced oscillations is studied in [11,12] up to first order in the strengths of sources of amplitude detuning, while ADeCTA requires higher order approximations. In the following we combine the approaches in [4] and $[11,12,30]$ to study the possible ADeCTA with forced oscillations.

The unperturbed Hamiltonian of a particle in an uncoupled lattice with a horizontal ac dipole is given by

$$
H_{0}=\frac{1}{2}\left[K_{1} x^{2}+K_{2} y^{2}+p_{x}^{2}+p_{y}^{2}+2 \delta(s, t) x\right],
$$

where $x, y, p_{x}, p_{y}$ are the canonical variables, $K_{1,2}$ are the normal focusing components and $\delta(s, t)$ is the time dependent kick from the ac dipole given by

$$
\delta(s, t)=\frac{q B L}{p} \delta_{\mathrm{dirac}}\left(s-s_{D}\right) \cos \left(2 \pi Q_{D} t+\psi_{D}\right),
$$

where $B L$ is the integrated field amplitude, $q$ is the charge of the particle, $p$ its momentum, $s_{D}$ the location, $Q_{D}$ is the driven tune, and $\psi_{D}$ is the initial phase of the ac dipole. The general equations of motion are given by $[30,31]$

$$
\begin{aligned}
& x=a_{1} u(\theta) e^{i Q_{H} \theta}+\bar{a}_{1} \bar{u}(\theta) e^{-i Q_{H} \theta}+\sqrt{2 \beta_{u}^{\prime} A_{1}} \cos \left(\phi_{u}^{\prime}\right), \\
& y=a_{2} v(\theta) e^{i Q_{V} \theta}+\bar{a}_{2} \bar{v}(\theta) e^{-i Q_{V} \theta}+\sqrt{2 \beta_{v}^{\prime} A_{2}} \cos \left(\phi_{v}^{\prime}\right),
\end{aligned}
$$

where $u, v$ and $\bar{u}, \bar{v}$ are the Floquet functions and their complex conjugates, respectively. $\beta_{u, v}^{\prime}$ and $\phi_{u, v}^{\prime}$ are the forced beta functions and phase advances as presented in [31]. $a_{1,2}, \bar{a}_{1,2}$ and $A_{1,2}$ are constants of motion. $A_{1,2}$ are determined by ac dipole parameters. When the ac dipole excitation tune is sufficiently close to the natural tune $A_{1,2}$ are approximated by [32]

$$
\sqrt{2 A_{1,2}}=\sqrt{\beta_{D}}\left|\frac{B L_{1,2}}{\left(B_{0} \rho\right)} \frac{e^{-i\left(\pi Q_{-}-\psi_{D}\right)}}{4 \sin \left(\pi Q_{-}\right)}\right|,
$$

where $\beta_{D}$ is the beta function at the ac dipole, $B L_{1,2}$ is the integrated ac dipole field amplitude, $\left(B_{0} \rho\right)$ is the magnetic rigidity, $Q_{-}$is the distance between the natural and ac dipole tunes, $Q_{-}=Q_{D}-Q_{H, V}$, and $\psi_{D}$ is the initial phase of the ac dipole.

The perturbations considered here are linear transverse coupling $K x y$ and the octupole cross term $K_{3} x^{2} y^{2}$, leaving the perturbing Hamiltonian as

$$
U=K x y+K_{3} x^{2} y^{2}
$$

These are the same terms that were shown to generate ADeCTA in [4].

Following [30] the equations of motion in presence of the perturbed Hamiltonian are derived using the former constants of motion as new variables as follows:

$$
\begin{aligned}
\frac{d a_{1}}{d \theta} & =i \frac{\partial U}{\partial \bar{a}_{1}} \\
\frac{\mathrm{d} \bar{a}_{1}}{d \theta} & =-i \frac{\partial U}{\partial a_{1}} \\
\frac{d a_{2}}{d \theta} & =i \frac{\partial U}{\partial \bar{a}_{2}} \\
\frac{\mathrm{d} \bar{a}_{2}}{d \theta} & =-i \frac{\partial U}{\partial a_{2}} .
\end{aligned}
$$

Expressing $U$ as function of the new variables and neglecting the rapid oscillating terms yields

$U=\bar{\kappa} a_{2} \overline{a_{1}} e^{-i \Delta \theta}+\kappa a_{1} \overline{a_{2}} e^{i \Delta \theta}+h_{u} a_{1} \overline{a_{1}} A_{2}+h_{v} a_{2} \overline{a_{2}} A_{1}$,

where

$$
\kappa=\frac{1}{4 \pi r} \int_{0}^{2 \pi} K_{s} \sqrt{\beta_{u} \beta_{v}} e^{i\left[\left(\phi_{u}-\phi_{v}\right)-\Delta \Theta\right]} d \Theta,
$$

where $r$ is the radius of the machine, $K_{s}$ is the skew quadrupolar strength, $\beta_{u, v}$ are the $\beta$ functions, $\phi_{u, v}$ are the phases, $\Theta=\frac{s}{r}$ and $\mathrm{s}$ is the position along the accelerator, $\Delta=Q_{H}-Q_{V}-p$ and $\bar{\kappa}$ is the complex conjugate [30]. $\Delta$ is the distance to the difference resonance, which we assume to be small so the $U$ above contains only slow varying terms. $h_{u, v}$ are defined as

$$
h_{u}=\frac{1}{2 \pi} \int_{0}^{2 \pi} K_{3} \beta_{u} \beta_{v}^{\prime} d \theta
$$




$$
h_{v}=\frac{1}{2 \pi} \int_{0}^{2 \pi} K_{3} \beta_{v} \beta_{u}^{\prime} d \theta
$$

where, actually, $h_{u, v}$ are almost identical to Eq. (2) with the difference that $h_{1111}$ does not depend on the forced $\beta^{\prime}$ functions. Feeding Eq. (8) with the perturbing Hamiltonian in Eq. (9) gives

$$
\begin{aligned}
& \frac{d a_{1}}{d \theta}=i \bar{\kappa} a_{2} e^{-i \Delta \theta}+i h_{u} a_{1} A_{2}, \\
& \frac{d a_{2}}{d \theta}=i \kappa a_{1} e^{i \Delta \theta}+i h_{v} a_{2} A_{1} .
\end{aligned}
$$

Defining new variables $\hat{a}_{1,2}$ as

$$
\begin{aligned}
& a_{1}=e^{i h_{u} A_{2} \theta} \hat{a}_{1}, \\
& a_{2}=e^{i h_{v} A_{1} \theta} \hat{a}_{2},
\end{aligned}
$$

Eq. (13) becomes

$$
\begin{aligned}
& \frac{\mathrm{d} \hat{a}_{1}}{d \theta}=i \bar{\kappa} \hat{a}_{2} e^{-i \hat{\Delta} \theta}, \\
& \frac{\mathrm{d} \hat{a}_{2}}{d \theta}=i \kappa \hat{a}_{1} e^{i \hat{\Delta} \theta},
\end{aligned}
$$

where $\hat{\Delta}$ has been defined as

$$
\hat{\Delta}=Q_{H}+h_{u} A_{2}-Q_{V}-h_{v} A_{1}-p
$$

and represents the new effective distance to the difference coupling resonance after including the amplitude detuning induced by the forced oscillations. The general solution of these coupled differential equations follows as in [30],

$$
\begin{aligned}
& \hat{a}_{1}=\bar{\kappa}\left(\frac{A_{+}}{w_{+}} e^{i w_{+} \theta}+\frac{A_{-}}{w_{-}} e^{i w_{-} \theta}\right), \\
& \hat{a}_{2}=\left(A_{+} e^{i w_{+} \theta}+A_{-} e^{i w_{-} \theta}\right) e^{i \theta \hat{\Delta}},
\end{aligned}
$$

where $A_{ \pm}$are complex constants of motion and $w_{ \pm}$are the frequencies given by

$$
w_{ \pm}=-\frac{\hat{\Delta}}{2} \pm \sqrt{\left(\frac{\hat{\Delta}}{2}\right)^{2}+|\kappa|^{2}} .
$$

$2|\kappa|$ is therefore the minimum separation between the two frequencies and it is independent of the amplitude of the forced motion. Therefore, contrary to free motion, forced motion with coupling $(K x y)$ and octupole terms $\left(K_{3} x^{2} y^{2}\right)$ does not feature an ADeCTA.

\section{B. Measurements and simulations}

In order to validate the theoretical results both measurement and simulations were done. The ac dipole

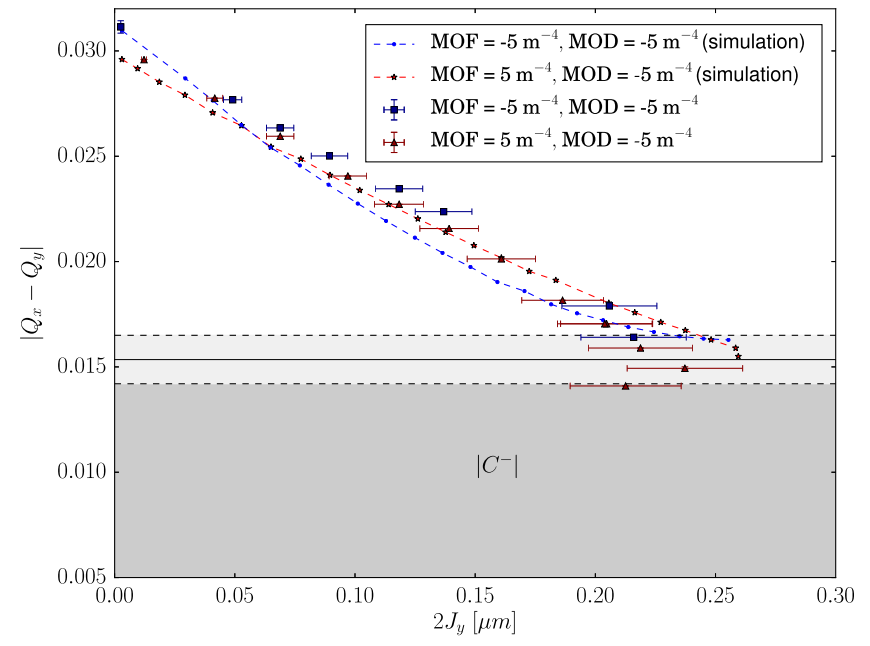

FIG. 2. The difference between the fractional horizontal and vertical tune as a function of vertical action for driven oscillations. The excitation was done using the ac dipole and the settings of the octupoles were: $\mathrm{MOF}=-5 \mathrm{~m}^{-4}, \mathrm{MOD}=$ $-5 \mathrm{~m}^{-4}$ and $\mathrm{MOF}=5 \mathrm{~m}^{-4}, \mathrm{MOD}=-5 \mathrm{~m}^{-4}$. The natural tunes were $Q_{x}=0.28$ and $Q_{y}=0.31$ and the drive tunes were $Q_{x, D}=$ 0.268 and $Q_{y, D}=0.32$. The dark gray area shows the linear $\left|C^{-}\right|$ and the light gray area show the uncertainty of the measured coupling.

excitations were applied for the two configurations of octupoles, described in Sec. II. The natural horizontal and vertical tunes were kept at $Q_{x}=0.28, Q_{y}=0.31$ and the driven tunes were $Q_{x, D}=0.268, Q_{y_{, D}}=0.32$. The available BPMs recorded 6600 turns of turn-by-turn data which corresponds to the period the beam is excited at a constant amplitude. The natural tune was obtained from the turn-by-turn spectra using sussix. The simulation aimed to reproduce the setup and an ac dipole with the same setting as for the experiment was used in the MAD-X tracking. The action was calculated based on the amplitude of the main driven tune and the $\beta$ functions at the BPM [33]. The amplitude of the horizontal excitation was increased in both simulation and during the measurement while the vertical excitation amplitude was kept small and constant. The results for the tune splits are shown in Fig. 2. The direct term for amplitude detuning from octupoles measured with ac dipole has been demonstrated to be a factor 2 larger than with free kicks, while the cross terms are only modified by the difference between the normal and driven $\beta$ functions, as seen in Eq. (2) [11]. It is therefore according to the expectation to see a larger detuning for the same action compared to the free kicks. We can also observe that we see slightly larger detuning for the case with same polarity. This is due to the fact that the opposite polarity reduces the cross terms and hence reduces the detuning. The simulations and the measurements are in very good agreement and the largest deviation is for the driven oscillations with the same polarity. In this case it seems that the simulations give a slightly larger detuning compared to the measurements. 
Tune drifts in the order of $10^{-3}$ between the two set of measurements would explain this. The model used does not include any errors and the purpose of the comparison is not to have a perfect agreement but to show that a simple model can accurately reproduce the measurements.

\section{CONCLUSION}

A compensation scheme for ADeCTA, generated by skew quadrupolar and normal octupolar fields, has for the first time been demonstrated in the LHC. The results are in qualitative agreement with previous theoretical understanding as well as with simulations. This could provide a way to mitigate this potential harmful modification of the Landau damping.

Analytical studies have shown that skew quadrupoles together with the octupole term: $K_{3} x^{2} y^{2}$ do not generate ADeCTA for forced oscillations. This higher order effect deviates from the established relations between free and forced amplitude detuning at first order. This has been confirmed with measurements and simulations that do not show any tendency to deviate away from the linear $\left|C^{-}\right|$. The fact that forced oscillations do not feature the ADeCTA as in free motion makes it difficult to measure this effect at configurations where fast reinjection is not possible, such as at collision energy in the LHC.

\section{ACKNOWLEDGMENTS}

The authors also would like to thank Massimo Giovannozzi for providing valuable feedback on the manuscript.

[1] E. H. Maclean, R. Tomás, F. Schmidt, and T. H. B. Persson, Measurement of nonlinear observables in the Large Hadron Collider using kicked beams, Phys. Rev. ST Accel. Beams 17, 081002 (2014).

[2] T. Persson, E. H. Maclean, and R. Tomás, Nonlinear coupling studies in the LHC, in Proceedings of 6th International Particle Accelerator Conference (IPAC'15), Richmond, VA, USA, 2015 (JACoW, Geneva, Switzerland, 2015), pp. 2105-2107, ISBN: 978-3-95450-168-7.

[3] O. Bruning, P. Collier, P. Lebrun, S. Myers, R. Ostojic, J. Poole, and P. Proudlock, CERN LHC Design Report No. CERN-2004-003-V1, 2004.

[4] R. Tomás, T. H. B. Persson, and E. H. Maclean, Amplitude dependent closest tune approach, Phys. Rev. Accel. Beams 19, 071003 (2016).

[5] P. Brüning, P. Collier, S. Lebrun, R. Myers, J. Ostojic, and P. Proudlock Poole, CERN LHC Design Report No. CERN-2004-003-V1, 2004.

[6] R. Tomás, Adiabaticity of the ramping process of an ac dipole, Phys. Rev. ST Accel. Beams 8, 024401 (2005).

[7] R. Tomás, R. Calaga, A. Langner, Y. I. Levinsen, E. H. Maclean, T. H. B. Persson, P. K. Skowronski, M. Stzelczyk, G. Vanbavinckhove, and R. Miyamoto, Record low beta beating in the LHC, Phys. Rev. ST Accel. Beams 15, 091001 (2012).

[8] T. Persson, F. Carlier, J. Coello de Portugal, A. GarciaTabares Valdivieso, A. Langner, E. H. Maclean, L. Malina, P. Skowronski, B. Salvant, R. Tomás, and A. C. Garcia Bonilla, LHC optics commissioning: A journey towards 1\% optics control, Phys. Rev. Accel. Beams 20, 061002 (2017).

[9] R. Miyamoto, Diagnostics of the Fermilab Tevatron using an ac dipole, Ph.D. thesis, Texas University, 2008.

[10] R. Tomás, M. Bai, R. Calaga, W. Fischer, A. Franchi, and G. Rumolo, Measurement of global and local resonance terms, Phys. Rev. ST Accel. Beams 8, 024001 (2005).

[11] S. White, E. Maclean, and R. Tomás, Direct amplitude detuning measurement with ac dipole, Phys. Rev. ST Accel. Beams 16, 071002 (2013).

[12] R. Tomás, X. Buffat, S. White, J. Barranco, P. Gonçalves Jorge, and T. Pieloni, Beam-beam amplitude detuning with forced oscillations, Phys. Rev. Accel. Beams 20, 101002 (2017).

[13] L. R. Carver et al., Instabilities and beam induced heating in 2015, in Proceedings, 6th Evian Workshop on LHC beam operation: Evian Les Bains, France, 2015 (CERN, Geneva, 2016), pp. 95-100.

[14] L. R. Carver, X. Buffat, K. Li, E. Metral, and M. Schenk, Transverse beam instabilities in the presence of linear coupling in the Large Hadron Collider, Phys. Rev. Accel. Beams 21, 044401 (2018).

[15] E. Métral, G. Hoffstaetter, and F. Willeke, Destabilizing effect of linear coupling in the HERA proton ring, in Proceedings, 8th European Conference, EPAC 2002, Paris, France, 2002 (2002), pp. 1535-1537, http:// weblib.cern.ch/abstract?CERN-PS-2002-021-AE.

[16] T. H. B. Persson and R. Tomás, Improved control of the betatron coupling in the Large Hadron Collider, Phys. Rev. ST Accel. Beams 17, 051004 (2014).

[17] T. H. B. Persson, Y.I. Levinsen, R. Tomás, and E. H. Maclean, Chromatic coupling correction in the Large Hadron Collider, Phys. Rev. ST Accel. Beams 16, 081003 (2013).

[18] T. Persson, G. Baud, J. Coello de Portugal, M. Gasior, M. Giovannozzi, J. Olexa, R. Tomás, A. Garcia-Tabares Valdivieso, and D. Valuch, Linear coupling dependence on intensity and a next step towards a feedback (MD1850), Report No. CERN-ACC-2017-0008, 2017.

[19] T. Persson, M. Gasior, A. Langner, T. Lefevre, E. H. Maclean, L. Malina, J. Olexa, J. Maria Coello de Portugal, P. Skowronski, R. Tomás, and A. GarciaTabares Valdivieso, Experience with DOROS BPMs for coupling measurement and correction, in Proceedings, 7th International Particle Accelerator Conference (IPAC 2016), Busan, Korea, 2016 (JACoW, Geneva, Switzerland, 2016), ISBN: 978-3-95450-147-2.

[20] E. Maclean, F. Carlier, S. Fartoukh, T. Persson, P. Skowronski, R. Tomás, and D. Wierichs, Demonstration of coupling correction below the per-mil limit in the LH, CERN Report No. CERN-ACC-NOTE-2016-0053, 2016.

[21] D. Pellegrini, F. Antoniou, S. Fartoukh, G. Iadarola, and Y. Papaphilippou, Multiparametric response of the LHC 
dynamic aperture in presence of beam-beam effects, J. Phys. Conf. Ser. 874, 012006 (2017).

[22] E. H. Maclean, T. H. B. Persson, and R. Tomás, Amplitude dependent closest tune approach generated by normal and skew octupoles, in Proceedings, 8th International Particle Accelerator, Conference (IPAC 2017): Copenhagen, Denmark, 2017 (JACoW, Geneva, Switzerland, 2017), ISBN: 978-3-95450-182-3.

[23] R. A. Barlow, E. Carlier, J. P. Pianfetti, V. Senaj, and M. Cattin, Control of the MKQA tuning and aperture kickers of the LHC, Report No. CERN-TE-Note-2010-001.

[24] R. Calaga and R. Tomas, Statistical analysis of RHIC beam position monitors performance, Phys. Rev. ST Accel. Beams 7, 042801 (2004).

[25] R. Bartolini and F. Schmidt, SUSSIX: A computer code for frequency analysis of nonlinear betatron motion, Report No. CERN SL/Note 98-017 (AP), 1998.

[26] T. Persson, F. Carlier, M. Gasior, E. H. Maclean, J. Olexa, R. Tomás, D. Valuch, and D. Wierichs, Suppression of amplitude dependent closest tune approach and first tests of the ADT as an ac-dipole (MD 1412), Report No. CERNACC-NOTE-2016-0057, 2016.
[27] R. Wolf, Field error naming conventions for LHC magnets, Report No. LHC-M-ES-0001 rev, https://lhc-div-mms.web .cern.ch/lhc-div-mms/tests/MAG/FiDeL/Documentation/ lhc-m-es-0001-30-00.pdf.

[28] http://mad.web.cern.ch/mad/.

[29] E. Forest, F Schmidt, and E. McIntosh, Introduction to the polymorphic tracking code, Report No. CERN-SL-2002044, 2002, https://cds.cern.ch/record/573082/files/CERNSL-2002-044-AP.pdf.

[30] G. Guignard, The general theory of all sum and difference resonances in a three-dimensional magnetic field in a synchrotron, Report No. CERN ISR-MA/75-23, 1975.

[31] R. Miyamoto, S. E. Kopp, A. Jansson, and M. J. Syphers, Parametrization of the driven betatron oscillation, Phys. Rev. ST Accel. Beams 11, 084002 (2008).

[32] R. Tomás, Normal form of particle motion under the influence of an ac dipole, Phys. Rev. ST Accel. Beams 5, 054001 (2002).

[33] F. S. Carlier, R. Tomas, E. H. Maclean, and T. H. B. Persson, First experimental demonstration of forced dynamic aperture measurements with LHC ac dipoles, Phys. Rev. Accel. Beams 22, 031002 (2019). 